\title{
Cunhantã Digital: programa de incentivo à participação de mulheres da região amazônica na Computação e áreas afins
}

\author{
Tanara Lauschner, Rosiane de Freitas, Fabíola Nakamura, Ludymila Lobo \\ ${ }^{1}$ Instituto de Computação - Universidade Federal do Amazonas (UFAM) \\ Manaus - AM - Brasil \\ \{tanara, rosiane, fabiola,llag\} @icomp.ufam.edu.br
}

\begin{abstract}
Resumo. Este artigo apresenta o Programa Cunhantã Digital, que envolve ações e projetos voltados ao incentivo à participação de mulheres da região amazônica na área de ciência e tecnologia. Tal programa contempla atividades como a realização de palestras e atividades lúdicas em eventos científicos e a capacitação de alunas para participar de competições de programação e desenvolver aplicativos computacionais, dentre outras. Algumas ações já resultaram em destaques obtidos em competições de programação e prêmios obtidos por meio do desenvolvimento de aplicativos, além de terem proporcionado um ambiente acadêmico mais propício à discussão de questões relacionadas ao gênero feminino nas áreas de computação e exatas na comunidade amazonense. Os próximos passos visam atingir também escolas de ensino fundamental e médio, para que as mulheres se sintam motivadas a escolher suas profissões independentemente dos pré-conceitos sociais.
\end{abstract}

\begin{abstract}
This article presents the Cunhantã Digital program involving actions and projects aimed at encouraging the participation of women in Science and Technology in the Amazon region. This program includes activities such as lectures at scientific meetings, training students to participate in competitions and develop computer applications. These actions resulted in highlights obtained in programming contests and prizes obtained through application development, and have provided a more conducive academic environment to discuss issues related to the female gender in the science and technology fields. The next steps are also intended to include primary and secondary schools so that women feel motivated to choose your career regardless of social prejudices.
\end{abstract}

\section{Introdução}

Estatisticamente, o percentual de mulheres nas áreas de Computação no Brasil é expressivamente menor que o de homens, representando uma das menores porcentagens de mulheres quando comparado a todos os outros cursos universitários [IPEA 2011]. Diante deste cenário, os estudos mostram diferentes motivos que podem ser apontados como causas para a disparidade entre a proporção de gêneros nestas áreas.

Alguns estudos apontam sexismo e cultura negativa como as principais causas de afastamento de mulheres de áreas tecnológicas [Hanton 2015]. O sexismo seria um fator que causa um ambiente não favorável à adesão e permanência de mulheres no setor. Já a 
cultura negativa, refere-se aos costumes sociais que vão desde o tipo de atividade lúdica realizada na infância até a expectativa da sociedade, e acabam ocasionando o desinteresse pela área, já que fatores biológicos não podem ser apontados como causa. Outros estudos [Medeiros e Borges 2014], a partir de análises em conversas de blogs de tecnologia, apontam o estereótipo negativo como sendo um dos principais fatores que repelem as mulheres nesta área.

Outro aspecto que pode ser considerado como desestimulante às alunas novatas na graduação é o grau de inibição em questionar o professor em sala de aula, conforme apresentado por Sales et. al. [Sales et al. 2014] em uma amostragem na qual $61 \%$ das alunas correspondeu à categoria "muito inibido", contra $29 \%$ do total em alunos. Complementando este fator, a pesquisa também demonstrou maior ausência de experiências anteriores relacionadas à lógica e à programação, na qual $37 \%$ dos homens já possuíam o conhecimento prévio, em contraste com apenas $17 \%$ do total de mulheres. Sendo assim, o grau de dificuldade apontado pelas mulheres foi maior que o dos homens cursando a mesma disciplina de Computação. A falta de incentivo na infância e adolescência das meninas a jogos e brinquedos que estimulam a lógica e/ou experiência em programação também é apontado como um fator que influencia nesta dificuldade inicial. Este estudo, apresentado na National Science Foundation (NSF), conclui que justamente na era em que o computador passou a ser pessoal, a frequência de acesso aos computadores passou a ser predominantemente masculina, devido ao marketing realizado na época ser direcionado aos homens, associando o universo tecnológico como algo voltado ao público masculino, fator que gerava desinteresse na maioria das mulheres. Assim, a NSF também aponta que a falta de conhecimento anterior à realização do curso de computação era um fator prejudicial.

Tendo em vista a ocorrência de tais problemas, a literatura apresenta diversas propostas para a solução, como a promoção de movimentos que visam a inserção de mulheres na área da computação realizado por lideranças do país, instituições de ensino e empresas [IPEA 2011], a propagação da ampla discussão sobre o assunto e estudos de medidas sobre como reverter o cenário [Medeiros and Borges 2014]. Para contribuição com tal causa, foi proposto o programa Cunhantã Digital, abordado neste trabalho, baseado em casos de grande impacto positivo proporcionado por ações voltadas para mulheres no desenvolvimento de aplicativos e participações em competições de programação científicas e tecnológicas [de Freitas et al. 2016] [MSR 2016], e na participação de ações nacionais da Sociedade Brasileira de Computação, por meio do Workshop Women In Technology (WIT) e o projeto Meninas Digitais [Maciel and Bim 2016].

O restante do artigo está estruturado como segue. Na Seção 2 são apresentados alguns programas e ações pelo Brasil e mundo, relacionados ao mesmo tema. Na Seção 3 são abordados projetos, ações e casos de sucesso do Programa Cunhantã Digital. E, na Seção 4 são feitas as considerações finais, na qual se resume os resultados e repercussão obtidos pelo programa, bem como as próximas ações a serem executadas.

\section{Ações e programas no Brasil e pelo mundo}

Existe um movimento mundial para promover uma maior participação de mulheres em áreas de ciências e tecnologia ou STEM (Science, Tecnology, Engineering and Mathematics), na qual as principais sociedades científicas possuem setores específicos para ações 
e estudo de tais questões, como a ACM-W (ACMW supporting, celebrating and advocating for Women in Computing) [ACM-W 2016], IEEE-W (IEEE Women in Engineering (WIE)) [IEEE-W 2016] e NCWIT (National Center for Women Information Technology) [NCWIT 2016]. A principal conferência científca especialmente sobre o tema é a Grace Hopper Celebration of Women in Computing [GHC 2016].

No Brasil, existe o Programa Meninas Digitais da Sociedade Brasileira de Computação [Maciel and Bim 2016], que tem como objetivo divulgar a área de Computação para despertar o interesse de estudantes do ensino médio/tecnológico ou dos anos finais do ensino fundamental, para que conheçam melhor a área e, desta forma, motivá-las a seguir carreira em Computação. Através deste programa, são realizados projetos como Meninas Digitais, MD-MT e elas++ [Maciel and Bim 2016]. A principal conferência científica sobre o tema no Brasil é o Women In Technology (WIT) realizado como parte do principal congresso de Computação do Brasil, o CSBC (Congresso da Sociedade Brasileira de Computação),e que está em sua 10a ediçao neste ano de 2016. Para a América Latina, há o Latin American Women in Computing Congress (LAWCC), evento satélite do CLEI (Latin American Computing Conference), um evento mais recente e que também está crescendo cada vez mais.

\section{Sobre o programa Cunhantã Digital e suas ações}

O Programa Cunhantã Digital sedimenta no Amazonas este movimento mundial crescente envolvendo ações para um maior envolvimento da mulher em áreas de ciência e tecnológica. Foi criado após várias iniciativas de sucesso envolvendo mulheres, em especial, em competições de programação científica e tecnológicas, bem como no desenvolvimento de aplicativos, como parte do Projeto SciTechGirls [de Freitas et al. 2016]. Nas subseções a seguir, será apresentado as ações de divulgação e conscientização realizadas por meio deste programa, tais como workshop e fóruns.

\subsection{Workshop Cunhantã Digital}

O Workshop Cunhantã Digital teve sua primeira edição em 21 de agosto de 2015, com duração de 6 horas úteis, cujo objetivo consistiu em estimular a maior participação das mulheres da região amazônica em áreas de ciência e tecnologia, promover a interação entre mulheres profissionais e meninas em formação, bem como estimular o ingresso de mulheres em cursos de graduação e carreiras de tais áreas. Teve a participação de mais de 200 pessoas, a maioria mulheres estudantes ou profissionais, mas também, para grande surpresa, contou com o interesse e participação de um número significativo de homens, representando $22 \%$ do total, que buscaram se inteirar mais desta problemática feminina e discutir questões de gênero na academia e mercado de trabalho. Houve palestras ministradas por pesquisadoras convidadas, dinâmicas de grupo, apresentação de aplicativos e casos de sucesso de algumas alunas de destaque e, também, um debate envolvendo mulheres de diversos segmentos, como política, indústria e academia. Uma nova edição está prevista para o segundo semestre deste ano de 2016.

\subsection{Fórum Cunhantã Digital}

Com base no sucesso do Workshop, foi realizado em janeiro deste ano de 2016 o I Fórum do Cunhantã Digital, voltado para educadores, de tal modo a se discutir e planejar ações 
para definição e planejamento de atividades a serem realizadas nas escolas do ensino fundamental e médio, bem como nos cursos universitários. O evento contou com muitos educadores da rede estadual e municipal de ensino, e das principais universidades da capital Manaus, e de alguns outros municípios do Amazonas, como Itacoatiara, Parintins, Benjamim Constant e Coari. Também contou a Superintendente da Zona Frahca de Manaus (SUFRAMA), órgão máximo para gestão das políticas e fomentos do Pólo Industrial do Amazonas, o que demonstra o prestígio alcançado pelo movimento Cunhantã Digital e o seu potencial de crescimento e fortalecimento de suas ações. Além do evento em si, é importante registrar o grande interesse da imprensa local pelo tema.

\subsection{Histórias de sucesso e formas de engajamento}

Apesar do pouco tempo de criação, já houve alguns casos de sucesso envolvendo alunas participantes de projetos e ações do Programa Cunhantã Digital, que são descritos em seguida.

Na Maratona de Programação 2015, promovida no Brasil pela SBC e mundialmente pela ACM - ICPC [MP 2016] [ICPC-ACM 2016], uma equipe do Instituto de Computação (IComp) da UFAM (Universidade Federal do Amazonas), composta somente por mulheres, se classificou para as finais brasileiras da competição, sendo a de melhor desempenho dentre as 08 (oito) equipes formadas somente por mulheres, em um total de mais de 600 equipes (com enorme predominância de homens). Também, algumas alunas individualmente se destacaram em diversas competições e eventos científicos. Houve a participação com destaque das 3 (três) únicas edições do International Women's Hackathon promovida pela Microsoft Research [MSR 2016], entre 2013 e 2014, culminando com o desenvolvimento de aplicativos de temática feminina: Mommy's BeneFIT, Make UPhi, What the Hack e How To Help.

\section{Considerações finais}

O Programa Cunhantã Digital tem sido muito bem recebido pela comunidade amazonense, já sendo observado um impacto muito positivo e que envolve educadores, profissionais e estudantes em torno da temática voltada para o aumento da participação feminina em cursos e profissões das áreas de ciência e tecnológica, com especial ênfase às áreas de Computação. Um canal de interação entre os educadores e profissionais promotores do programa e os jovens estudantes interessados no movimento (a maioria mulheres, mas, também, muitos homens) tem sido mantido via rede social Facebook [Lauschner et al. 2016]. Como atividades em andamento, tem sido realizadas competições de programação e sendo promovidas atividades envolvendo o desenvolvimento de aplicativos [de Freitas et al. 2016]. Como próximos passos, já está prevista a realização do II Workshop e do II Fórum do programa Cunhantã Digital, bem como a realização de um ciclo de palestras a serem ministradas por alunas universitárias e profissionais da área em escolas do ensino médio e fundamental. Também houve um convite para o programa ser apresentado na 7a. Feira Norte do Estudante, que costuma receber mais de dez mil estudantes do ensino fundamental e médio, na qual é oportunizado aos alunos conhecerem o curso que desejam escolher, e onde com isto se espera motivar também as meninas estudantes a conhecerem mais os cursos e carreiras de Computação e áreas afins, colaborando para o aumento do ingresso de meninas em cursos universitários destas áreas. 


\section{Referências}

ACM-W (2016). Acm-w supporting, celebrating and advocating for women in computing. Association for Computing Machinery (ACM). Disponível em https://women.acm.org/.

de Freitas, R., Conte, T., Lobo, L., and Salles, J. (2016). Projeto scitechgirls. Instituto de Computação, Universidade Federal do Amazonas. Disponível em http://scitechgirls.icomp.ufam.edu.br/.

GHC (2016). Grace hopper celebration of women in computing. Anita Borg Institute. Disponível em http://ghc.anitaborg.org/.

Hanton, P. B. (2015). The lack of women in technology: The role culture and sexism play. Master's thesis, Master's Capstone Theses.

ICPC-ACM (2016). International collegiate programming contest. Association for Computing Machinery (ACM). Disponível em https://icpc.baylor.edu/.

IEEE-W (2016). Ieee women in engineering (wie). Institute of Electrical and Electronics Engineers, (IEEE). Disponível em http://www.ieee.org/membership_services/membership/women/index.html.

IPEA (2011). Retrato das desigualdades de gênero e raça. Instituto de Pesquisa Econômica Aplicada. 4a. edição.

Lauschner, T., Nakamura, F., de Freitas, R., Conte, T., Lobo, L., and Cruz, M. (2016). Programa cunhantã digital. Instituto de Computação, Universidade Federal do Amazonas. Disponível em https://www.facebook.com/cunhantadigital/?fref=ts.

Maciel, C. and Bim, S. A. (2016). Programa meninas digitais - ações para divulgar a computação para meninas do ensino médio. In Anais do Computer on the Beach. Sociedade Brasileira de Computação. Disponível em http://www.computeronthebeach.com.br/arquivos-2016/Anais completos - Computer on the Beach 2016.pdf.

Medeiros, C. and Borges, J. (2014). Abram-se Às mulheres todas as portas!": Conversas em blogs de mulheres em carreira de ti. Em Blogs De Mulheres Em Carreira De TI. Gestão Contemporânea.

MP (2016). Maratona de programação. The ICPC-ACM South America - Brazil. Sociedade Brasileira de Computação. Disponível em http://maratona.ime.usp.br/.

MSR (2016). Microsoft research. Disponível em http://research.microsoft.com/.

NCWIT (2016). National center for women and information technology. Disponível em https://www.ncwit.org/.

Sales, A., Calado, B., Silva, D., Mattos, G., and Moreira, J. (2014). Dificuldades para o ingresso e permanência na ciência e engenharia da computação: Um olhar feminino. $18^{\circ}$ Redor - Universidade Federal Rural de Pernambuco - Recife. 